\title{
STABLE BUNDLES ON POSITIVE PRINCIPAL ELLIPTIC FIBRATIONS
}

\author{
Misha Verbitsky
}

\begin{abstract}
Let $\stackrel{\pi}{\longrightarrow} X$ be a principal elliptic fibration over a Kähler base $X$. We assume that the Kähler form on $X$ is lifted to an exact form on $M$ (such fibrations are called positive). Examples of these are regular Vaisman manifolds (in particular, the regular Hopf manifolds) and Calabi-Eckmann manifolds. Assume that $\operatorname{dim} M>2$. Using the Kobayashi-Hitchin correspondence, we prove that all stable bundles on $M$ are flat on the fibers of the elliptic fibration. This is used to show that all stable vector bundles on $M$ take form $L \otimes \pi^{*} B_{0}$, where $B_{0}$ is a stable bundle on $X$, and $L$ a holomorphic line bundle. For $X$ algebraic this implies that all holomorphic bundles on $M$ are filtrable (that is, obtained by successive extensions of rank-1 sheaves). We also show that all positive-dimensional compact subvarieties of $M$ are pullbacks of complex subvarieties on $X$.
\end{abstract}

\section{Introduction}

Let $M$ be a compact complex manifold, and $M \stackrel{\pi}{\longrightarrow} X$ a smooth holomorphic fibration. Assume that an elliptic curve (considered as a complex Lie group) acts on $M$ holomorphically. Assume, moreover, that this action is free and transitive on the fibers of $\pi$. Then $\pi: M \longrightarrow X$ is called a principal elliptic fibration. The fibers of $\pi$ are identified (non-canonically) with $T$.

This terminology is somewhat misleading, as in algebraic geometry one says "elliptic fibraion" speaking of a fibration with a section. However, a principal bundle with a section is trivial, hence we use (following [BM2], [BM1]) the term "principal elliptic fibration" used to describe something which should be more properly called "a 1-dimensional torus principal bundle".

In physics, the same object is usually called "a $T^{2}$-bundle". There is a great body of literature studying these manifolds in physical context, see e.g. [CCD], [GP] and the references therein. One usually considers a principal elliptic fibraion over a 2-dimensional Calabi-Yau manifold, equipped with a Hermitian metric, such that its Bismut connection has holonomy $S U(3) .{ }^{1}$ Such manifolds appear as a background for the heterotic string in the presence of fluxes.

Received March 31, 2004.

Misha Verbitsky is an EPSRC advanced fellow supported by CRDF grant RM1-2354-MO02 and EPSRC grant GR/R77773/01.

${ }^{1}$ Bismut connection on $M$ is a Hermitian connection in $T M$, with totally antisymmetric torsion; it is not difficult to show that such connection is unique on any Hermitian complex manifold. 
Examples of principal elliptic fibrations are very common in complex geometry (take, for instance, the Hopf surface $\mathbb{C}^{2} \backslash 0 /\langle q\rangle$, fibered over $\mathbb{C} P^{1}$ with a fiber $\mathbb{C}^{*} /\langle q\rangle$, where $\left.q \in \mathbb{C},|q|>1\right)$. For more examples see Subsection 2.1. The Hopf surface $M$ is clearly homeomorphic to $S^{1} \times S^{3}$. Since $H^{2}(M)=0$, it is not Kähler. This is a general phenomenon. Unless $\pi$ is trivialized after taking a finite cover, the total space $M$ of a principal elliptic fibration is not Kähler.

In this paper we are interested in the so-called positive principal elliptic fibrations (Definition 2.1). These are the fibrations with the Kähler base $X$, such that some Kähler form $\omega_{X}$ on $X$ is lifted to an exact form on $M$. Heuristically this means that the fibration $M \stackrel{\pi}{\longrightarrow} X$ is positive in the sense of complex algebraic geometry (see Remark 2.4 for a more detailed explanation). From the positivity one obtains that $M$ admits an exact positive 2 -form $\pi^{*} \omega_{X}$ with all eigenvalues strictly positive except one.

In [Ve], [OV2] such form was used in Vaisman geometry to obtain Kodaira-type embedding theorem, several Kodaira-Nakano-type vanishing results and a study of compact subvarieties of a Vaisman manifold. We apply a similar reasoning to positive elliptic fibrations. Using the Kobayashi-Hitchin correspondence (Subsection 3.2), we obtain the following theorem.

Theorem 1.1: Let $M \stackrel{\pi}{\longrightarrow} X, \operatorname{dim}_{\mathbb{C}} M=n$ be a positive principal elliptic fibration, $(B, \nabla)$ a Hermitian-Einstein bundle on $M$, and $\Theta \in \Lambda^{1,1}(M) \otimes \operatorname{End}(B)$ its curvature. Assume that $n \geqslant 3$. Then $\Theta(v, \cdot)=0$ for any vertival tangent vector $v \in T_{\pi} M$.

Proof. This is Theorem 4.3.

For a definition of stability and Hermitian-Einstein connections see Section 3. Theorem 1.1 implies the following corollary.

Proposition 1.2: Let $T$ be an elliptic curve, and $M \stackrel{\pi}{\longrightarrow} X$ a positive principal $T$-fibration, equipped with a preferred Hermitian metric. The universal covering $\tilde{T}$ acts on $M$ in a standard way (its action is factorized through $T$ ). Consider a stable bundle $B$ on $M$. Then $B$ is equipped with a natural holomorphic $\tilde{T}$ equivariant structure.

Proof. This is Proposition 4.6.

A similar argument proves

Proposition 1.3: Let $M \stackrel{\pi}{\longrightarrow} X$ be a positive principal elliptic fibration, and $Z \subset M$ a closed positive-dimensional subvariety. Then $Z=\pi^{*}\left(Z_{0}\right)$ for some complex subvariety $Z_{0} \subset X$.

Proof. This is Proposition 4.5. 
Applying the $\tilde{T}$-equivariant structure arising on stable bundles, the following structure theorem is obtained

Theorem 1.4: Let $M \stackrel{\pi}{\longrightarrow} X$ be a positive principal elliptic fibration equipped with a preferred Hermitian metric, and $B$ a stable holomorphic bundle on $M$. Then $B \cong L \otimes \pi^{*} B_{0}$, where $L$ is a line bundle on $M$ and $B_{0}$ a stable bundle on $X$.

Proof. This is Theorem 6.1.

We also show that all coherent sheaves on $M$ are filtrable, that is, obtained as successive extensions of rank 1 sheaves (Theorem 6.5).

\section{Principal elliptic fibrations}

2.1. Positive elliptic fibrations: definition and examples. Throughout this paper, $M$ is a compact complex manifold, $X$ a Kähler manifold, $T$ an elliptic curve, and $M \stackrel{\pi}{\longrightarrow} X$ a principal $T$-fibration

Definition 2.1: A fibration is called positive if the pullback $\pi^{*} \omega_{X}$ is exact, for some Kähler form $\omega_{X}$ on $X$.

Principal toric fibrations, their invariants, topology and Dolbeault cohomology are thoroughly analyzed in the excellent paper [Hö]. For our purposes, the most important examples are the following.

Example 2.2: Regular Vaisman manifolds are principal elliptic fibrations constructed the following way (see e.g. [DO], [OV2]). Take a projective manifold $X$, and let $L$ be an ample line bundle on $X$. Consider the space $\tilde{M}:=\operatorname{Tot}\left(L^{*} \backslash 0\right)$, which is a total space of the dual bundle $L^{*}$ without the zero section. Then $\tilde{M}$ is a principal $\mathbb{C}^{*}$-bundle over $X$. Fix $q \in \mathbb{C},|q|>1$, and let $M:=\tilde{M} / \sim_{q}$, be the quotient of $\tilde{M}$ under the equivalence $v \sim q v, v \in L^{*} \backslash 0$. Then $M$ is a principal elliptic bundle, with a fiber $T=\mathbb{C}^{*} /\langle q\rangle$. The positivity of $M$ is elementary (see e.g. [Ve] or [OV2]).

Vaisman manifolds were studied in great detail by I. Vaisman (see e.g. [Va1]), under the name of generalized Hopf manifolds.

Remark 2.3: A special case of the above example is a regular Hopf manifold $M \cong S^{2 n-1} \times S^{1}$, obtained as a quotient of $\mathbb{C}^{n} \backslash 0$ under an equivalence generated by $v \longrightarrow q v$. Clearly, $M$ is fibered over $\mathbb{C} P^{n-1}$, with a fiber $T=\mathbb{C}^{*} /\langle q\rangle$. Regular Hopf manifold is obtained if one applies the construction of Example 2.2 to $X=\mathbb{C} P^{n-1}, L=\mathcal{O}(1)$. 
Remark 2.4: Taking an arbitrary line bundle $L$ on a Kähler manifold $X$ and applying the same construction as above, we obtain a principal toric bundle as well. It will be positive in the sense of Definition 2.1 if and only if the bundle $L$ is positive, as one can easily see from the exact sequence associated with the fibration in [Hö]

$$
H^{1}(T) \stackrel{\delta}{\longrightarrow} H^{2}(X) \stackrel{i}{\longrightarrow} H^{2}(M) .
$$

The second arrow of (2.1) is a standard pullback map, and the transgression $\delta$ maps one of the generators of $H^{1}(T)$ to zero, the other to $c_{1}(L)$.

Example 2.5: The Calabi-Eckmann manifolds $M \cong S^{2 n+1} \times S^{2 m+1}$ are fibered in standard way over $\mathbb{C} P^{n} \times \mathbb{C} P^{m}$ (the spheres $S^{2 n+1}, S^{2 m+1}$ are fibered over $\mathbb{C} P^{n}, \mathbb{C} P^{m}$ by the way of the Hopf fibration). The complex structure on $M$ is constructed as follows. Fix a number $\tau \in \mathbb{C} \backslash \mathbb{R}$. Consider the action of an additive group $\mathbb{C}$ on $\left.\mathbb{C}^{n+1} \backslash 0\right) \times\left(\mathbb{C}^{m+1} \backslash 0\right.$,

$$
t\left(v_{1}, v_{2}\right) \longrightarrow\left(e^{t} v_{1}, e^{t \tau} v_{2}\right)
$$

This action is clearly holomorphic, and the quotient space $M$ is naturally identified with $S^{2 n+1} \times S^{2 m+1} ;^{1}$ moreover, $M$ is equipped with a natural holomorphic projection $M \stackrel{\pi}{\longrightarrow} \mathbb{C} P^{n} \times \mathbb{C} P^{m}$. The fibers of $\pi$ are identified with $\mathbb{C} /\langle 1, \tau\rangle$. It is not difficult to see that $M$ is a principal elliptic fibration. Since $H^{2}(M)=0$, $M$ is obviously positive.

2.2. Preferred Hermitian metrics. Let $M \stackrel{\pi}{\longrightarrow} X$ be a principal elliptic fibration. As always, we assume that $M$ is compact. By Blanchard's Theorem, unless $\pi$ is trivialized on a finite covering, $M$ is non-Kähler (see e.g. [Hö]). If $M$ is positive, the pullback of a Kähler metric becomes exact, hence $\pi$ cannot be trivialized. Therefore, a positive principal elliptic fibration is never Kähler.

We shall always assume that $M$ is equipped with a special kind of Hermitian metric, defined as follows.

Recall that the smooth surjective map $\pi:(M, g) \longrightarrow\left(X, g_{X}\right)$ of Riemannian manifolds is called Riemannian submersion if its differential $d \pi$ induces an isometry $d \pi: T_{\pi} M^{\perp} \longrightarrow T X$, where $T_{\pi} M$ denotes the space of vertical tangent vectors, and $T_{\pi} M^{\perp}$ is orthogonal complement.

Definition 2.6: Let $T$ be an elliptic curve, and $M \stackrel{\pi}{\longrightarrow} X$ a positive principal $T$-fibration. Consider a Kähler metric $g_{X}$ on $X$, such that the pullback $\pi^{*} \omega_{X}$ of the corresponding Kähler form is exact. A Hermitian metric $g$ on $M$ is called preferred if $g$ is $T$-invariant, and the projection $\pi:(M, g) \longrightarrow\left(X, g_{X}\right)$ is a Riemannian submersion.

\footnotetext{
${ }^{1}$ To see this isomorphism, take a Hermitian metric on $\mathbb{C}^{n+1}, \mathbb{C}^{m+1}$. Then $\left|e^{t} v_{1}\right|=\left|e^{t \tau} v_{2}\right|=$ 1 if and only if $\operatorname{Re}(t)=\log \left|v_{1}\right|, \operatorname{Re}(\tau t)=\log \left|v_{2}\right|$. Since $\tau \notin \mathbb{R}$, such $t$ exists and is clearly unique.
} 
Clearly, preferred Hermitian metrics always exist.

Remark 2.7: Let $M$ be a regular Vaisman manifold from Example 2.2, and $\tau \in T M$ the real vector field which is tangent to the map $v \longrightarrow \lambda v, \lambda \in \mathbb{R}$, $v \in L^{*}$. Then $M$ admits a Hermitian metric $g$ for which $\tau$ acts by isometries, and, moreover, $\nabla_{L C} \tau=0$ for the Levi-Civita connection associated with this metric. In terminology used in Vaisman geometry, $g$ is called the locally conformally Kähler Gauduchon metric, and $\tau$ the Lee field ([DO]). From the condition $\nabla_{L C} \tau=0$ one immediately obtains that $\pi:(M, g) \longrightarrow\left(X, g_{X}\right)$ is a Riemannian submersion (see e.g. [OV1]). By construction, $\tau$ is a generator of the Lie algebra of $T$. Since $\tau$ acts by isometries, $g$ is $T$-invariant. We obtain that the locally conformally Kähler Gauduchon metric on a regular Vaisman manifold is a preferred one.

\section{Stable bundle on Hermitian manifolds}

In this section, we recall the necessary definitions and explain the KobayashiHitchin correspondence on non-Kähler manifolds. We follow [LT1] and [LT2].

\subsection{Gauduchon metrics and stability.}

Definition 3.1: Let $(M, g)$ be a Hermitian manifold, and $\omega \in \Lambda^{1,1}(M)$ its Hermitian form. We say that $g$ is a Gauduchon metric if $\partial^{*} \bar{\partial}^{*} \omega=0$, or, equivalently, $\partial \bar{\partial}\left(\omega^{\operatorname{dim}_{\mathbb{C}} M-1}\right)=0$.

Recall that the metrics $g, g^{\prime}$ on $M$ are called conformally equivalent if $g=f g^{\prime}, f \in C^{\infty} M$.

Theorem 3.2: ([Ga]) Let $(M, g)$ be a compact Hermitian manifold. Then there exists a unique Gauduchon metric $g^{\prime}$ which is conformally equivalent to $g$.

Definition 3.3: Let $M$ be a compact complex manifold equipped with a Gauduchon metric, and $\omega \in \Lambda^{1,1}(M)$ the corresponding Hermitian form. Consider a torsion-free coherent sheaf $F$ on $M$. Denote by $\operatorname{det} F$ its determinant bundle. Pick a Hermitian metric $\nu$ on $\operatorname{det} F$, and let $\Theta$ be the curvature of the associated Chern connection. We define the degree of $F$ as follows:

$$
\operatorname{deg} F:=\int_{M} \Theta \wedge \omega^{\operatorname{dim}_{\mathbb{C}} M-1} .
$$

This notion is independent from the choice of the metric $\nu$. Indeed, if $\nu^{\prime}=e^{\psi} \nu$, $\psi \in C^{\infty}(M)$, then the associated curvature form is written as $\Theta^{\prime}=\Theta+\partial \bar{\partial} \psi$, and

$$
\int_{M} \partial \bar{\partial} \psi \wedge \omega^{\operatorname{dim}_{\mathbb{C}} M-1}=0
$$

because $\omega$ is Gauduchon. 
If $F$ is a Hermitian vector bundle, $\Theta_{F}$ its curvature, and the metric $\nu$ is induced from $F$, then $\Theta=\operatorname{Tr}_{F} \Theta_{F}$. In Kähler case this allows one to relate degree of a bundle with the first Chern class. However, in non-Kähler case, the degree is not a topological invariant - it depends fundamentally on the holomorphic geometry of $F$. Moreover, the degree is not discrete, as in the Kähler situation, but takes values in continuum.

Further on, we shall see that one can in some cases construct a holomorphic structure of any given degree $\lambda \in \mathbb{R}$ on a fixed $C^{\infty}$-bundle. In our examples, such holomorphic structures are constructed on a topologically trivial line bundle over a positive principal elliptic fibration (Remark 5.4).

Definition 3.4: Let $F$ be a non-zero torsion-free coherent sheaf on $M$. Then slope $(F)$ is defined as

$$
\operatorname{slope}(F):=\frac{\operatorname{deg} F}{\operatorname{rk} F}
$$

The sheaf $F$ is called

$$
\begin{array}{ll}
\text { stable } & \text { if for all subsheaves } F^{\prime} \subset F \text {, we have slope }\left(F^{\prime}\right)<\operatorname{slope}(F) \\
\text { semistable } & \text { if for all subsheaves } F^{\prime} \subset F \text {, we have slope }\left(F^{\prime}\right) \leqslant \operatorname{slope}(F) \\
\text { polystable } & \text { if } F \text { can be represented as a direct sum of stable } \\
& \text { coherent sheaves with the same slope. }
\end{array}
$$

Remark 3.5: This definition is stability is "good" as most standard properties of stable and semistable bundles hold in this situation as well. In particular, all line bundles are stable; all stable sheaves are simple; the Jordan-Hölder and Harder-Narasimhan filtrations are well defined and behave in the same way as they do in the usual Kähler situation ([LT1], [Br]).

However, not all bundles are filtrable, that is, are obtained as successive extensions by coherent sheaves of rank 1 . There are non-filtrable vector bundles e.g. on a non-algebraic K3 surface (Example 6.4).

3.2. Kobayashi-Hitchin correspondence. The statement of Kobayashi and Hitchin correspondence is translated to the Hermitian situation verbatim, following Li and Yau ([LY]).

Definition 3.6: Let $B$ be a holomorphic Hermitian vector bundle on a Hermitian manifold $M$, and $\Theta \in \Lambda^{1,1}(M) \otimes \operatorname{End}(B)$ the curvature of its Chern connection $\nabla$. Consider the operator $\Lambda: \Lambda^{1,1}(M) \otimes \operatorname{End}(B) \longrightarrow \operatorname{End}(B)$ which is a Hermitian adjoint to $b \longrightarrow \omega \otimes b, \omega$ being the Hermitian form on $M$. The connection $\nabla$ is called Hermitian-Einstein (or Yang-Mills) if $\Lambda \Theta=$ const $\cdot \operatorname{Id}_{B}$.

Theorem 3.7: (Kobayashi-Hitchin correspondence) Let $B$ be a holomorphic vector bundle on a compact complex manifold equipped with a Gauduchon metric. Then $B$ admits a Hermitian-Einstein connection $\nabla$ if and only if $B$ is polystable. Moreover, the Hermitian-Einstein connection is unique. 
Proof. See [LY], [LT1], [LT2].

Remark 3.8: The bundle $B$ is stable if and only if the corresponding HermitianEinstein bundle $(B, \nabla)$ cannot be decomposed onto a direct sum of sub-bundles. In this case, the Hermitian-Einstein metric is defined by the connection uniquely up to a constant multiplier.

\section{Hermitian-Einstein bundles on positive principal elliptic fibrations}

\subsection{Preferred metrics are Gauduchon.}

Proposition 4.1: Let $M \stackrel{\pi}{\longrightarrow} X$ be a positive principal elliptic fibration, and $g$ a preferred Hermitian metric. Then $g$ is Gauduchon.

Proof. Consider the orthogonal decomposition $T M=T_{\pi} M \oplus T_{\pi} M^{\perp}$ of the tangent bundle onto its vertical component and its orthogonal complement. We write the Hermitian form $\omega$ as $\omega=\omega_{0}+\omega_{T}$, where $\omega_{0}$ vanishes on $T_{\pi} M$ and $\omega_{T}$ vanishes on $T_{\pi} M^{\perp}$. Since $\pi$ is a Riemannian submersion, $\omega_{0}=\pi^{*} \omega_{X}$, where $\omega_{X}$ is a Kähler form on $X$. This implies

$$
\partial^{*} \bar{\partial}^{*} \omega_{0}=\pi^{*} \partial^{*} \bar{\partial}^{*} \omega_{X}=0 .
$$

On the other hand, $\partial^{*} \bar{\partial}^{*} \omega_{T}$ can be computed by restricting $\omega_{T}$ to the fibers $\pi^{-1}(x)$ of $\pi$ and computing $\partial^{*} \bar{\partial}^{*}\left(\left.\omega_{T}\right|_{\pi^{-1}(x)}\right)$. Since $\omega_{T}$ is $T$-invariant,

$$
\partial^{*} \bar{\partial}^{*}\left(\left.\omega_{T}\right|_{\pi^{-1}(x)}\right) \text { also vanishes, }
$$

and we have

$$
\partial^{*} \bar{\partial}^{*} \omega=\partial^{*} \bar{\partial}^{*} \omega_{0}+\partial^{*} \bar{\partial}^{*} \omega_{T}=0
$$

4.2. Primitive forms on positive principal elliptic fibrations. Let $B_{0}$ be a vector bundle with connection, and $\eta$ a $B_{0}$-valued form. Then $\eta$ is called closed if $\nabla \eta=0$, where

$$
\nabla: \Lambda^{i}(M) \otimes B \longrightarrow \Lambda^{i+1}(M) \otimes B
$$

is the connection operator extended to forms by the Leibniz rule. The curvature form of a connection is closed, by Bianchi identity.

Further on in this section, we shall need the following result.

Proposition 4.2: Let $M \stackrel{\pi}{\longrightarrow} X$ be a positive principal elliptic fibration, $n=$ $\operatorname{dim} M \geqslant 3$, equipped with a preferred Hermitian metric, $B$ a Hermitian bundle with connection, and $\Theta \in \Lambda^{1,1}(M) \otimes \operatorname{End}(B)$ a closed (1,1)-form. Assume that $\Theta$ is primitive, that is, $\Lambda \Theta=0$. Then $\Theta(v, \cdot)=0$ for any vertcal tangent vector $v \in T_{\pi} M$. 
Proof. Since the connection $\nabla$ is Hermitian, it preserves the natural real structure in $\Lambda^{1,1}(M) \otimes \operatorname{End}(B), \eta \otimes b \longrightarrow \bar{\eta} \otimes b^{\perp}$, where by $b^{\perp}$ one understands the Hermitian adjoint endomorphism. Therefore, we may assume that $\Theta$ is real, with respect to this real structure.

Let $\theta, \theta_{1}, \ldots, \theta_{n-1}$ be an orthonormal basis in $\Lambda^{1,0}(M)$, with $\theta \in T_{\pi} M, \theta_{i} \in$ $T_{\pi} M^{\perp}$. Consider a decomposition

$$
\begin{aligned}
\Theta & =\sum_{i \neq j}\left(\theta_{i} \wedge \bar{\theta}_{j}+\bar{\theta}_{i} \wedge \bar{\theta}_{j}\right) \otimes b_{i j}+\sum_{i}\left(\theta_{i} \wedge \bar{\theta}_{i}\right) \otimes a_{i} \\
& +\sum_{i}\left(\theta \wedge \bar{\theta}_{i}+\bar{\theta} \wedge \bar{\theta}_{i}\right) \otimes b_{i}+\theta \wedge \bar{\theta} \otimes a
\end{aligned}
$$

with $b_{i j}, b_{i}, a_{i}, a \in \mathfrak{u}(B)$ being skew-Hermitian endomorphisms of $B$.

Consider now the form $\omega_{0}:=\pi^{*} \omega_{X}$. This form is exact, positive, and has $n-1$ strictly positive eigenvalues. Using the basis described above, we can write

$$
\omega=\sqrt{-1}\left(\theta \wedge \bar{\theta}+\sum_{i} \theta_{i} \wedge \bar{\theta}_{i}\right), \quad \omega_{0}=\sqrt{-1}\left(\sum_{i} \theta_{i} \wedge \bar{\theta}_{i}\right)
$$

where $\omega$ is the Hermitian form of $M$. This follows directly from $\omega$ being a preferred Hermitian form.

Let $\Xi:=\operatorname{Tr}(\Theta \wedge \Theta)$. This is a closed (2,2)-form on $M$. Then (4.1) implies

$$
(\sqrt{-1})^{n} \Xi \wedge \omega_{0}^{n-2}=\operatorname{Tr}\left(-\sum b_{i}^{2}+a\left(\sum a_{i}\right)\right)
$$

On the other hand, $\sum a_{i}+a=\Lambda \Theta=0$, hence

$$
(\sqrt{-1})^{n} \Xi \wedge \omega_{0}^{n-2}=\operatorname{Tr}\left(-\sum b_{i}^{2}-a^{2}\right) .
$$

Since $\operatorname{Tr}\left(-a^{2}\right)$ is a positive definite form on $\mathfrak{u}(B)$, the integral

$$
\int_{M}(\sqrt{-1})^{n} \Xi \wedge \omega_{0}^{n-2}
$$

is non-negative, and positive unless $b_{i}$ and $a$ both vanish everywhere. Using $n>2$, we find that (4.3) vanishes, because $\omega_{0}$ is exact and $\Xi$ is closed. Therefore, $b_{i}$ and $a$ are identically zero, which is exactly the claim of Proposition 4.2

\subsection{The Lübke-type positivity for Hermitian-Einstein bundles.}

Theorem 4.3: Let $M \stackrel{\pi}{\longrightarrow} X, \operatorname{dim}_{\mathbb{C}} M=n$ be a positive principal elliptic fibration, equipped with a preferred Hermitian metric, $(B, \nabla)$ a Hermitian-Einstein bundle on $M$, and $\Theta \in \Lambda^{1,1}(M) \otimes \operatorname{End}(B)$ its curvature. Assume that $n \geqslant 3$. Then $\Theta(v, \cdot)=0$ for any vertical tangent vector $v \in T_{\pi} M$.

Proof. Theorem 4.3 is proven by the standard positivity argument, going back to M. Lübke $([\mathrm{Lü}])$. Similar argument is used e.g. to show that any HermitianEinstein bundle with vanishing Chern classes is flat.

Let $\Theta_{0}:=\Theta-\frac{1}{\mathrm{rk} F} \operatorname{Tr} \Theta$ be the traceless part of $\Theta$. Then $\Theta_{0}$ is primitive and closed, hence, by Proposition $4.2, \Theta_{0}(v, \cdot)=0$ for all $v \in T_{\pi} M$. To prove 
Theorem 4.3, it remains to show that the 2-form $\operatorname{Tr} \Theta$ also vanishes on $v \in T_{\pi} M$. This is implied by the following trivial lemma.

Lemma 4.4: Let $M \stackrel{\pi}{\longrightarrow} X$, $\operatorname{dim}_{\mathbb{C}} M \geqslant 3$ be a positive principal elliptic fibration, equipped with a preferred Hermitian metric, and $\eta \in \Lambda^{1,1}(M)$ a closed form satisfying $\Lambda \eta=$ const. Then $\eta(v, \cdot)=0$ for all $v \in T_{\pi} M$.

Proof. Clearly, $\Lambda \omega_{0}=$ const. Replacing $\eta$ with $\eta-c \omega_{0}$, we may assume that $\Lambda \eta=0$. Now $\eta$ is primitive, and, by Proposition $4.2, \eta(v, \cdot)=0$ for all $v \in T_{\pi} M$. This finishes the proof of Theorem 4.3.

4.4. Complex subvarieties in positive principal elliptic fibrations. A similar argument proves the following result.

Proposition 4.5: Let $M \stackrel{\pi}{\longrightarrow} X$ be a positive principal elliptic fibration, and $Z \subset M$ a closed positive-dimensional subvariety. Then $Z=\pi^{*}\left(Z_{0}\right)$ for some complex subvariety $Z_{0} \subset X$.

Proof. The proof is taken from [Ve] verbatim, where the same result was proven for Vaisman manifolds.

It is well known that

$$
\int_{Z} \omega_{0}^{k} \geqslant 0
$$

for all complex subvarieties $Z \subset M, \operatorname{dim}_{\mathbb{C}} Z=k$, and all positive forms $\omega_{0}$. Moreover, the integral (4.4) vanishes if and only if $Z$ is tangent to the null-space foliation of $\omega_{0}$.

Since $\omega_{0}$ is exact, the integral (4.4) vanishes. Therefore, $Z$ is tangent to the null-space foliation of $\omega_{0}$. This implies $Z$ is tangent to the fibers of $\pi$.

\subsection{Equivariance of stable bundles.}

Theorem 4.3 has an immediate implication for the holomorphic geometry of stable bundles.

Proposition 4.6: Let $T$ be an elliptic curve, and $M \stackrel{\pi}{\longrightarrow} X, \operatorname{dim}_{\mathbb{C}} M \geqslant 3$ a positive principal $T$-fibration, equipped with a preferred Hermitian metric. The universal covering $\tilde{T}$ acts on $M$ in a standard way (its action is factorized through $T$ ). Consider a stable bundle $B$ on $M$. Then $B$ is equipped with a natural holomorphic $\tilde{T}$-equivariant structure.

Proof. Let $\nabla$ be a Hermitian-Einstein connection on $B$, and $\tau$ a holomorphic vector field tangent to the action of $T$. Consider the operator $\nabla_{\tau}: B \longrightarrow B$. Since the curvature of $B$ vanishes on $\tau, \nabla_{\tau}$ is holomorphic. For any $\lambda \in \mathbb{C}$ the operator $e^{\lambda \nabla_{\tau}}$ of parallel translation along $\tau$ defines a holomorphic $\tilde{T}$-equivariant structure on $B$. 
Remark 4.7: The same argument implies that a stable bundle on a Vaisman manifold is always equivariant with respect to the action of the Lee field (see e.g. [DO], [OV2] for details).

\section{Line bundles on principal elliptic fibrations}

Let $T$ be an elliptic curve and $M \stackrel{\pi}{\longrightarrow} X$ a positive principal $T$-fibration, equipped with a preferred Hermitian metric. Any line bundle $L$ on $M$ is stable. From Proposition 4.6 we obtain a natural $\tilde{T}$-equivariant structure on $L$, and from Theorem 4.3 a connection $\nabla$ which is flat on the fibers of $\pi$. Monodromy of this connection is related to the $\tilde{T}$-equivariant structure as follows. Let $\Gamma$ be the kernel of the natural projection $\tilde{T} \longrightarrow T, \Gamma=\pi_{1} T \cong \mathbb{Z}^{2}$. Using the $\tilde{T}$ equivariant structure, we find that $\Gamma$ acts on $L$ by automorphisms. This action coincides with the monodromy of $\nabla$ along the fibers of $\pi$.

The group of holomorphic automorphisms of $L$ is identified with $\mathbb{C}^{*}$, therefore the monodromy map acts as a character $\chi: \Gamma \longrightarrow \mathbb{C}^{*}$. Since $\Gamma$ is a monodromy of a Hermitian connection, $\chi$ takes values in $U(1)$. We denote this character by $\chi_{L}: \Gamma \longrightarrow U(1)$.

It turns out that any character can be realized by some line bundle. The following proposition is the main result of this section.

Proposition 5.1: Let $T$ be an elliptic curve and $M \stackrel{\pi}{\longrightarrow} X$ a positive principal $T$-fibration, $\operatorname{dim} M \geqslant 3$. Consider any character $\chi: \Gamma \longrightarrow U(1)$. Then there exists a holomorphic line bundle $L$ on $M$ such that the corresponding character $\chi_{L}: \Gamma \longrightarrow U(1)$ is equal to $\chi$.

Proof. Consider the commutative diagram with exact rows coming from the exponential exact sequence

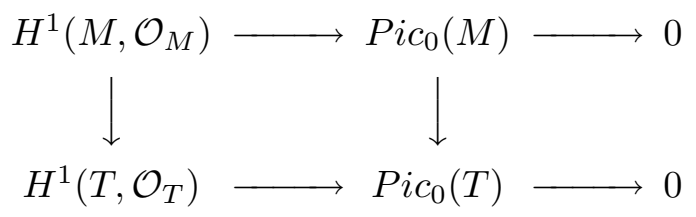

The characters $\chi: \Gamma \longrightarrow U(1)$ are in bijective correspondence with the bundles from $\mathrm{Pic}_{0}(T)$, and the correspondence is provided by a unique flat connection on every $L \in P i c_{0}(T)$ (this follows e.g. from the Kobayashi-Hitchin correspondence on elliptic curce). Therefore, to prove Proposition 5.1 it is sufficient and necessary to show that the natural arrow $\mathrm{Pic}_{0}(M) \longrightarrow P i c_{0}(T)$ is surjective. As one can see from (5.1), this is implied by surjectivity of the natural restriction map

$$
H^{1}\left(M, \mathcal{O}_{M}\right) \longrightarrow H^{1}\left(T, \mathcal{O}_{T}\right)
$$


Since $\operatorname{dim} H^{1}\left(T, \mathcal{O}_{T}\right)=1$, it is actually sufficient to show that (5.2) is non-trivial. Looking again on (5.1), we find that to show non-triviality of (5.2) it is sufficient to prove that $\operatorname{Pic}_{0}(M) \longrightarrow \operatorname{Pic}_{0}(T)$ is non-trivial.

We reduced Proposition 5.1 to the following lemma

Lemma 5.2: In assumptions of Proposition 5.1, there exists a holomorphic line bundle $L$ on $M, L \in P i c_{0}(M)$ such that $L$ is non-trivial on fibers of $\pi: M \longrightarrow X$.

Remark 5.3: Restriction of $L$ to different fibers of $\pi$ is a flat bundle with the monodromy determined by the character $\chi_{L}$. Therefore, the restriction $\left.L\right|_{\pi^{-1}(x)}$ is independent from the choice of $x \in X$ (the fibers of $\pi$ are naturally identified because the $\pi$ is a principal fibration).

Proof of Lemma 5.2: Consider a trivial line bundle $L_{\text {triv }}$ on $M$ with trivial flat connection $\nabla_{\text {triv }}$. Let $\theta$ be a 1 -form which satisfies $d \theta=\omega_{0}$, where $\omega_{0}=\pi^{*} \omega_{X}$ is the pullback of the Kähler form on $X$.

Consider the connection $\nabla_{0}=\nabla_{\text {triv }}+\theta$ on $L_{\text {triv }}$. By definition, the curvature of $\nabla_{0}$ is equal $\omega_{0}$. Therefore, $\left(\nabla_{0}^{0,1}\right)^{2}=0$, and $\nabla_{0}$ defines a holomorphic structure on $L_{\text {triv }}$. Denote the corresponding holomorphic line bundle by $L_{0}$. The degree of $L_{0}$ is easy to compute:

$$
\operatorname{deg} L_{0}=\int_{M} \omega \wedge \omega_{0}^{n-1}
$$

and this number is clearly positive. Therefore, $L_{0}$ is a non-trivial holomorphic bundle. Lemma 5.2 is proven. We proved Proposition 5.1.

Remark 5.4: Let $q \in \mathbb{R}$ be a number. Consider the connection $\nabla^{\prime}=\nabla_{\text {triv }}+q \theta$ on $L_{\text {triv }}$. Clearly, its curvature is equal $q \omega_{0}$. Therefore, $\nabla^{\prime}$ defines a holomorphic structure on $L_{\text {triv }}$. Denote the corresponding holomorphic bundle by $L(q)$. From (5.3) we obtain that $\operatorname{deg} L(q)=q \operatorname{deg} L$. Therefore, the trivial $C^{\infty}$-bundle $L_{\text {triv }}$ admits holomorphic structures of any given degree.

\section{Structure theorem for stable bundles}

6.1. Equivariant $\tilde{T}$-action and the stable bundles. The main result of this paper is the following theorem.

Theorem 6.1: Let $M \stackrel{\pi}{\longrightarrow} X, \operatorname{dim}_{\mathbb{C}} M \geqslant 3$ be a positive principal elliptic fibration equipped with a preferred Hermitian metric, and $B$ a stable holomorphic bundle on $M$. Then $B \cong L \otimes \pi^{*} B_{0}$, where $L$ is a line bundle on $M$ and $B_{0}$ a stable bundle on $X$.

Proof. As Proposition 4.6 implies, $B$ is $\tilde{T}$-equivariant. The kernel $\Gamma$ of the natural projection $\tilde{T} \longrightarrow T$ acts on $B$ by holomorphic automorphisms. Since $B$ is stable, all its automorphisms are proportional to identity. Therefore, $\Gamma$ acts 
on $B$ by characters $\chi: \Gamma \longrightarrow \mathbb{C}^{*}$. Since $\chi$ can be obtained via monodromy of the Hermitian-Einstein connection, $\chi$ takes values in $U(1)$. Let $L$ be a line bundle on $M$ which is $\tilde{T}$-equivariant and has the same monodromy action (such line bundle exists by Proposition 5.1). Then $B \otimes L^{-1}$ has trivial monodromy on the fibers of $\pi$. Clearly, $B \otimes L^{-1}=\pi^{*} B_{0}$ for some holomorphic bundle $B_{0}$ on $X$. Since $L$ is by construction Hermitian-Einstein, the same is true for $B \otimes L^{-1}$ and for $B_{0}$ (the last assertion is true because $\pi$ is a holomorphic Riemannian submersion). We proved Theorem 6.1.

Remark 6.2: In [BS] S. Bando and Y.-T. Siu developed Kobayashi-Hitchin correspondence for reflexive coherent sheaves. Using these results, it is easy to extend Theorem 6.1 for reflexive coherent sheaves. We obtain that any stable reflexive coherent sheaf $F$ on $M$ is isomorphic to $L \otimes \pi^{*} F_{0}$, where $F_{0}$ is a stable reflexive coherent sheaf on $X$.

\subsection{Filtrable coherent sheaves on positive elliptic fibrations.}

Definition 6.3: Let $Z$ be a complex variety. A coherent sheaf $F$ on $Z$ is called filtrable if and only if the following equivalent conditions are satisfied.

(i): $F$ is obtained as a successive extenstion of coherent sheaves of rank 1

(ii): $F$ admits a filtration

$$
0=F_{0} \subset F_{1} \subset F_{2} \ldots \subset F_{N}=F,
$$

with $\operatorname{rk}\left(F_{i} / F_{i-1}\right)=1$.

On a quasiprojective variety $Z$, every coherent sheaf is filtrable. This is not true if $Z$ is not algebraic, as the following example shows.

Example 6.4: Let $M$ be a K3 surface with $\operatorname{Pic}(M)=0$ (generic non-algebraic K3 surfaces have $\operatorname{Pic}(M)=0$ ). Since all line bundles on $M$ are trivial, no stable vector bundle $B, \operatorname{rk} B>1$ can be filtrable. However, the tangent bundle $T M$ is stable, because it is Hermitian-Einstein. Therefore, $T M$ is not filtrable.

Theorem 6.5: Let $M \stackrel{\pi}{\longrightarrow} X$ be a positive principal elliptic fibration, $\operatorname{dim} M \geqslant$ 3. Assume that $X$ is projective. Then all coherent sheaves on $M$ are filtrable.

Proof: Let $F$ be a coherent sheaf on $M$. Using the Harder-Narasimhan filtration $([\mathrm{Br}])$, we reduce Theorem 6.5 to the case when $F$ is semistable. Using the Jordan-Hölder filtration, we reduce it to the case when $F$ is a stable reflexive sheaf. By Theorem 6.1 (see Remark 6.2), $F=\pi^{*} F_{0} \otimes L$, where $F_{0}$ is a stable reflexive sheaf on $X$. Since $X$ is projective, $F_{0}$ is filtrable. Then $F$ is also filtrable. We proved Theorem 6.5. 


\section{Acknowledgements}

I am grateful to Ruxandra Moraru who explained to me the geometry of holomorphic bundles on elliptic fibrations and gave me reference I needed, and to Tony Pantev, who told me about the applications and physical background of this geometry. Tony also found an error in a preliminary version of this paper. My gratitude also to F. Bogomolov and D. Kaledin, who gave me important insights on the geometry of Bott-Samelson manifolds.

\section{References}

[BS] S. Bando, Y.-T. Siu, Stable sheaves and Einstein-Hermitian metrics, In: Geometry and Analysis on Complex Manifolds, Festschrift for Professor S. Kobayashi's 60th Birthday, ed. T. Mabuchi, J. Noguchi, T. Ochiai, World Scientific, (1994), 39-50.

[BM1] V. Brinzanescu, R. Moraru, Stable bundles on non-Kahler elliptic surfaces, math.AG/0306192, 15 pages.

[BM2] , Twisted Fourier-Mukai transforms and bundles on non-Kahler elliptic surfaces, math.AG/0309031, 13 pages.

[Br] L. Bruasse, Harder-Narasimhan filtration on non-Kähler manifolds, Int. Journal of Maths, 12 (2001), 579-594.

[CCD] G. L. Cardoso, G. Curio, G. Dall'Agata, D. Lüst, P. Manousselis and G. Zoupanos, Non-Kähler string backgrounds and their five torsion classes, 33 pages, hepth/0211118.

[DO] S. Dragomir, L. Ornea, Locally conformal Kähler geometry, Progress in Mathematics, 155. Birkhäuser, Boston, MA, 1998.

[Ga] P. Gauduchon, La 1-forme de torsion d'une variete hermitienne compacte, Math. Ann., 267 (1984), 495-518.

[GP] E. Goldstein anf S. Prokushkin, Geometric model for complex non-Kähler manifolds with $S U(3)$ structure, 21 pages, hep-th/0212307.

[Hö] T. Höfer, Remarks on torus principal bundles, J. Math. Kyoto Univ. 33 (1993), 227259.

[LY] J. Li, and S.-T. Yau, Hermitian Yang-Mills connections on non-Kahler manifolds, in "Mathematical aspects of string theory" (S.T. Yau ed.), World Scientific Publ., London, (1987), 560-573.

[Lü] M. Lübke, Chernklasses von Hermite-Einstein Vektorbündeln, Math. Ann. 260 (1982), 133-141.

[LT1] M. Lübke, A. Teleman, The Kobayashi-Hitchin correspondence, World Scientific Publishing Co., Inc., River Edge, NJ, 1995. x+254 pp

[LT2] , The universal Kobayashi-Hitchin correspondence on Hermitian manifolds, math.DG/0402341, 90 pages.

[M] R. Moraru, Integrable systems associated to a Hopf surface, Canad. J. Math. 55 (2003), 609-635.

[OV1] L. Ornea, M. Verbitsky, Structure theorem for compact Vaisman manifolds, math.DG/0305259, also in: Math. Res. Lett, 10 (2003), 799-805

[OV2] _ Immersion theorem for Vaisman manifolds, math.AG/0306077, 28 pages, to appear in Mathematische Annalen (DOI: 10.1007/s00208-004-0620-4).

[Va1] I. Vaisman, Generalized Hopf manifolds, Geom. Dedicata 13 (1982), 231-255.

[Ve] M. Verbitsky, Vanishing theorems for locally conformal hyperkähler manifolds, 2003, math.DG/0302219, also in Proc. of Steklov Institute, (2004), 54-79. 
University of Glasgow, Department of Mathematics, 15 University Gardens, GlasGOW G12 8QW, Scotland.

E-mail address: verbit@maths.gla.ac.uk

Institute of Theoretical and Experimental Physics, B. Cheremushininskaya, 25, Moscow, 117259, Russia

E-mail address: verbit@mccme.ru 